Article

\title{
Antimicrobial and Antibiofilm Activities of Weissella cibaria against Pathogens of Upper Respiratory Tract Infections
}

\author{
Ji-Eun Yeu ${ }^{1,2}$, Hyeon-Gyu Lee ${ }^{2}$, Geun-Yeong Park ${ }^{1}$, Jisun Lee ${ }^{3} \mathbb{D}$ and Mi-Sun Kang ${ }^{1, *(\mathbb{D})}$ \\ 1 R\&D Center, OraPharm, Inc., Seoul 04782, Korea; ji-eun85@orapharm.com (J.-E.Y.); \\ gypark@orapharm.com (G.-Y.P.) \\ 2 Department of Food and Nutrition, Hanyang University, Seoul 04763, Korea; hyeonlee@hanyang.ac.kr \\ 3 Bio-Healthcare Food Science Interdisciplinary Major, School of Humanities, Art \& Technology, \\ Kookmin University, Seoul 02707, Korea; health.livinglab@gmail.com \\ * Correspondence: jieenkang@orapharm.com
}

check for

updates

Citation: Yeu, J.-E.; Lee, H.-G.; Park,

G.-Y.; Lee, J.; Kang, M.-S.

Antimicrobial and Antibiofilm

Activities of Weissella cibaria against

Pathogens of Upper Respiratory Tract

Infections. Microorganisms 2021, 9,

1181. https://doi.org/10.3390/

microorganisms 9061181

Academic Editors: Haruki Kitazawa

and Julio Villena

Received: 17 May 2021

Accepted: 27 May 2021

Published: 30 May 2021

Publisher's Note: MDPI stays neutral with regard to jurisdictional claims in published maps and institutional affiliations.

Copyright: (c) 2021 by the authors. Licensee MDPI, Basel, Switzerland. This article is an open access article distributed under the terms and conditions of the Creative Commons Attribution (CC BY) license (https:// creativecommons.org/licenses/by/ $4.0 /)$.

\begin{abstract}
Recently discovered preventive effects of probiotics on oral health have attracted interest to their use for the prevention and treatment of various diseases. This study aimed to evaluate the antimicrobial and antibiofilm properties of Weissella cibaria against Streptococcus pyogenes, Staphylococcus aureus, S. pneumoniae, and Moraxella catarrhalis, the major pathogens of upper respiratory tract infections (URTIs). The antimicrobial activities of W. cibaria were compared with those of other oral probiotics using a competitive inhibition assay and the determination of the minimum inhibitory concentrations (MICs). In addition, a time-kill assay, spectrophotometry, and confocal laser scanning microscopy were used to confirm the antimicrobial and antibiofilm abilities of $W$. cibaria CMU (oraCMU) and CMS1 (oraCMS1). Both live cells and cell-free supernatants of all tested probiotics, except Streptococcus salivarius, showed excellent antimicrobial activities. All target pathogens were killed within 4 to $24 \mathrm{~h}$ at twice the MIC of oraCMU and oraCMS1, which showed the highest antimicrobial activities against $M$. catarrhalis. The antimicrobial substances that affected different target pathogens were different. Both oraCMU and oraCMS1 showed excellent abilities to inhibit biofilm formation and remove preformed biofilms. Our results suggest that the W. cibaria probiotics offer new possibilities for the prevention and treatment of bacterial URTIs.
\end{abstract}

Keywords: probiotic; antimicrobial; antibiofilm; upper respiratory tract; Weissella cibaria

\section{Introduction}

Upper respiratory tract infections (URTIs) are infections of the nose, sinuses, throat, larynx, and epiglottis [1]. Acute URTIs are primarily caused by viruses [2]. The most common viral URTI is nasopharyngitis (cold), which is experienced by adults $2-5$ times a year [3]. Acute URTIs have been reported to cause a high disease burden, accounting for $40 \%$ of the reasons for absenteeism among adult workers and for $10 \%$ of patients visiting outpatient and emergency rooms [4,5].

Acute URTIs are sometimes caused by bacteria. Streptococcus pyogenes, S. pneumoniae, Haemophilus influenzae, Moraxella catarrhalis, and Staph. aureus are common and important pathogens of bacterial infections [6-9]. In particular, S. pyogenes is the most clinically important bacterial agent for acute pharyngitis and acute pharyngeal tonsillitis [7].

In children between 6 months and 3 years of age, approximately $90 \%$ of acute otitis media are reported to be associated with viral URTIs. In particular, it has been reported that half of the children infected with specific upper respiratory pathogens, such as S. pneumoniae, H. influenzae, and M. catarrhalis, develop acute otitis media after viral URTIs [10].

Although acute URTIs are more frequently associated with viruses than with bacteria, viral infections usually heal spontaneously and rapidly improve. Meanwhile, the rate of improvement of bacterial infections is slow, and the risk of recurrence and chronicity 
increases as the infection progresses $[7,10]$. There is no specific treatment other than antibiotics for bacterial URTIs. However, the use of antibiotics has been associated with several problems, including poor patient compliance, allergies, unnecessary side effects, abuse, and tolerance [11,12]. In particular, biofilm-forming bacteria do not respond well to general antibiotic treatments. When biofilms are formed, bacteria have been reported to be 500 times more resistant to antibiotics [13]. Therefore, it is very important to control URTI-related bacteria.

In recent years, there has been increasing interest in the topical application of probiotics to prevent or treat diseases [14]. Several clinical studies have confirmed the effectiveness of probiotics for preventing URTIs. According to Altadill et al. [15], administration of probiotics has beneficial effects on URTIs by significantly reducing the number of days of a URTI and the rate of fever. Another clinical study has reported that the annual episodes of pharyngeal tonsillitis and the incidence of acute otitis media due to streptococcal infection were significantly reduced by the use of probiotics [16]. However, few studies have evaluated the in vitro effectiveness of oral care probiotics in controlling major pathogens of URTIs, which pose a high risk for recurrence and chronicity.

Weissella cibaria CMU (oraCMU) and CMS1 (oraCMS1) are oral care live probiotics that are used as commercial strains to aid oral health. These bacteria have been confirmed to be safe in experiments recommended by the Food and Agriculture Organization of the United Nations/World Health Organization and via other animal and human applications [17-19]. It has been reported that $W$. cibaria has a higher hydrogen peroxide $\left(\mathrm{H}_{2} \mathrm{O}_{2}\right)$ production potential than that of other commercial oral care probiotics and shows excellent antibacterial and antibiofilm effects against periodontal and dental caries bacteria in the oral cavity [20].

Therefore, in this study, W. cibaria was evaluated for its antibacterial activity against major pathogens of URTIs, as well as for the ability to inhibit pathogenic biofilm formation and facilitate the removal of biofilms.

\section{Materials and Methods}

\subsection{Bacterial Strains and Growth Conditions}

The four major pathogens of URTIs used in this study were S. pyogenes KCCM 40411, Staph. aureus KCTC 1928, S. pneumoniae ATCC 6303, and M. catarrhalis KCCM 42707. S. pyogenes KCCM 40411 and M. catarrhalis KCCM 42707 were purchased from the Korean Culture Center of Microorganisms (Seoul, Korea). Staph. aureus KCTC 1928 was purchased from the Korean Collection for Type Cultures (Daejeon, Korea). S. pneumoniae ATCC 6303 was provided by Chonnam National University (Gwangju, Korea). Five commercial oral care probiotics, including W. cibaria CMU (oraCMU), W. cibaria CMS1 (oraCMS1), Streptococcus salivarius, Ligilactobacillus salivarius, and Limosilactobacillus reuteri, were used in this study. The preparations of oraCMU and oraCMS1 were provided by OraPharm, Inc. (Seoul, Korea). S. salivarius was isolated from a commercial probiotic product using tryptic soy agar (Difco, Detroit, MI, USA). L. salivarius and L. reuteri were also isolated from commercial probiotic products using de Man, Rogosa, and Sharpe (MRS) agar (Difco). All bacterial strains were identified using $16 \mathrm{~S}$ rRNA sequence analysis. W. cibaria, L. salivarius and L. reuteri cultures were grown aerobically in MRS broth at $37^{\circ} \mathrm{C}$ for $16 \mathrm{~h}$. Streptococcus spp. and Staph. aureus cultures were grown aerobically in brain heart infusion (BHI) broth (Difco) at $37^{\circ} \mathrm{C}$ for 16 h. Staph. aureus was incubated with shaking, and S. pyogenes and S. pneumoniae were incubated under $5 \% \mathrm{CO}_{2}$ conditions. M. catarrhalis was cultured on $\mathrm{BHI}$ agar plates for 2 days at $37^{\circ} \mathrm{C}$ and $5 \% \mathrm{CO}_{2}$.

\subsection{Antimicrobial Activity}

2.2.1. Competitive Inhibition Assay

A competitive inhibition assay was used to compare the antimicrobial effects of the oral care probiotics on the growth of the target pathogens in coculture. The antimicrobial effects were determined based on the competitive index (CI), which was calculated as 
follows [21]: $\mathrm{CI}$ of the test pathogen $=$ [pathogen colony-forming units $(\mathrm{CFU}) /$ probiotic $\mathrm{CFU}$ at $16 \mathrm{~h}] /$ (pathogen $\mathrm{CFU} /$ probiotic CFU at $0 \mathrm{~h}$ ).

\subsubsection{Determination of Minimum Inhibitory Concentrations (MICs)}

MICs were determined using a microtiter plate assay [22]. Oral care probiotics were centrifuged at $5000 \times g$ for $10 \mathrm{~min}$ at $4{ }^{\circ} \mathrm{C}$ and filtered through a $0.45 \mu \mathrm{m}$ syringe filter to prepare cell-free supernatants (CFSs). The CFS of each oral care probiotic was serially diluted 2-fold to final concentrations of 31.25 to $500 \mathrm{mg} / \mathrm{mL}(v / v)$. In a 96-well plate, $100 \mu \mathrm{L}$ of the target pathogen (final concentration: $\sim 5 \times 10^{6} \mathrm{CFU} / \mathrm{mL}$ ) were added to wells containing $100 \mu \mathrm{L}$ of an oral care probiotic. Each plate included a positive control (target pathogen alone) and negative control (medium). After incubation at each growth condition, the growth of bacteria was determined by measuring the absorbance at $600 \mathrm{~nm}\left(\mathrm{OD}_{600}\right)$ using a microplate reader (VersaMax, Molecular Devices, San Jose, CA, USA).

\subsubsection{Time-Kill Assay}

Time-kill curves [23] were used to evaluate the bactericidal activities of the W. cibaria strains. CFSs of the W. cibaria strains were used at MIC and twice the MIC. The target pathogen (final concentration: $\sim 5 \times 10^{6} \mathrm{CFU} / \mathrm{mL}$ ) and each CFS were mixed equally in a 96-well plate. The plates were monitored for bacterial growth over different culture periods at different intervals based on preliminary assay results for each culture condition. Bacterial growth was determined by measuring the number of viable cells and $\mathrm{OD}_{600}$.

\subsubsection{Characterization of Antimicrobial Substances}

The effects of antimicrobial substances, such as organic acids, $\mathrm{H}_{2} \mathrm{O}_{2}$, and bacteriocinlike compounds (BLCs), were evaluated according to our previous study [24]. Briefly, to evaluate the effects of organic acids, CFSs of the W. cibaria strains were treated with proteinase K $(0.1 \mathrm{mg} / \mathrm{mL}$; Sigma-Aldrich, St. Louis, MO, USA) and catalase $(0.05 \mathrm{mg} / \mathrm{mL}$; Sigma-Aldrich). After neutralizing the CFS, proteinase K treatment was used to evaluate the effect of $\mathrm{H}_{2} \mathrm{O}_{2}$, and the neutralized CFS was treated with catalase to evaluate the effect of BLCs. The treated samples were serially diluted 2-fold, and $100 \mu \mathrm{L}$ were added to a 96-well plate. The target pathogen was adjusted to $\mathrm{OD}_{600}$ of 0.05 (final concentration: $\sim 5 \times 10^{6} \mathrm{CFU} / \mathrm{mL}$ ) with the growth medium, and $100 \mu \mathrm{L}$ were inoculated into each well. After incubation of the target pathogen under each condition, $\mathrm{OD}_{600}$ was measured using a microplate reader.

\subsection{Antibiofilm Formation Activity}

\subsubsection{Inhibition of Biofilm Formation}

To investigate the inhibitory abilities of live cells of the W. cibaria strains on pathogenic biofilm formation, coculture was performed using a Transwell insert (Corning, New York, NY, USA). The target pathogen was inoculated into a well of the lower compartment, and $W$. cibaria was inoculated into the upper part of the Transwell insert. The inoculum concentrations of both bacteria were the same (final concentration: $2.5 \times 10^{7} \mathrm{CFU} / \mathrm{mL}$ ). To evaluate the inhibitory abilities of CFSs of the W. cibaria strains on pathogenic biofilm formation, the pathogen and CFS were mixed equally in the wells. The target pathogen alone was used as a positive control. The amount of the biofilm formed in each well under each growth condition was measured by spectrophotometry after 24 and $48 \mathrm{~h}$. Briefly, at the end of each incubation period, the culture medium was removed, and after air-drying, each well was stained with $500 \mu \mathrm{L}$ of $1 \%$ crystal violet for $10 \mathrm{~min}$. The stain was removed, and the wells were washed three times with phosphate-buffered saline. One milliliter of absolute ethanol was added to each well to dissolve the stain absorbed by the biofilm. The dissolved stain was dispensed into 96-well plates, and the absorbance was measured at $595 \mathrm{~nm}$ using a microplate reader. 


\subsubsection{Removal of Preformed Biofilms}

To investigate whether live cells of the $W$. cibaria strains were able to remove preformed pathogenic biofilms, each target pathogen was incubated in the lower chamber of a Transwell insert for $24 \mathrm{~h}$ under growth conditions, followed by inoculation of the W. cibaria strains into the upper chamber of the Transwell insert and incubation for an additional 24 and $48 \mathrm{~h}$. The effects of CFSs of the $W$. cibaria strains were evaluated as described above. The target pathogen alone was used as a positive control. The residual amount of biofilm in the well was measured spectrophotometrically, as described above.

\subsubsection{Confocal Laser Scanning Microscopy (CLSM) Analysis}

To perform CLSM analysis of biofilm formation, a $12 \mathrm{~mm}$ diameter coverslip (SPL Life Sciences, Gyeonggi-do, Korea) was placed in a 24 -well plate, and culture was performed under the same experimental conditions as described above. The biofilm was gently washed with sterile saline and stained with the Filmtracer LIVE/DEAD biofilm viability kit (Thermo Fisher Diagnostics SpA, Rodano, Italy) according to the manufacturer's instructions. The stained biofilm was imaged at a $40 \times$ magnification using a confocal laser scanning microscope (Leica Microsystems, Wetzlar, Germany). Images were processed and analyzed using Las X (Leica Microsystems CMS GmbH, Mannheim, Germany) to evaluate the overall biofilm volume as an estimate of the total biomass and to calculate the live/dead cell ratio.

\subsection{Statistical Analysis}

The results are presented as the mean \pm standard deviation for triplicate measurements. Differences between the means were evaluated using one-way analysis of variance with Duncan's multiple range test. Differences were considered significant at $p<0.05$. All statistical tests were performed using SPSS Statistics version 21.0 for Windows (IBM Corp., Armonk, NY, USA).

\section{Results}

3.1. Antimicrobial Activity

\subsubsection{Competitive Inhibition}

CI values were calculated to evaluate competitive inhibition of the major pathogens of URTIs by the oral care probiotics (Figure 1). Except those obtained with S. salivarius, the CI values of the test pathogens were less than 1 . Most of the live probiotics, including the $W$. cibaria strains, showed excellent competitiveness against the pathogens, while $S$. salivarius had the lowest antimicrobial activity.

\subsubsection{MIC Results}

The antimicrobial activities of CFSs of the five oral care probiotics against the four major pathogens of URTIs were evaluated using MIC values (Table 1). The best antimicrobial activity against S. pyogenes was shown by the CFS of L. salivarius with a MIC of $125 \mathrm{mg} / \mathrm{mL}$. All strains, except S. salivarius, showed the same MICs of $125 \mathrm{mg} / \mathrm{mL}$ for Staph. aureus, S. pneumoniae, and M. catarrhalis. The CFS of S. salivarius did not show antimicrobial activity against any of the target pathogens. 
(a)

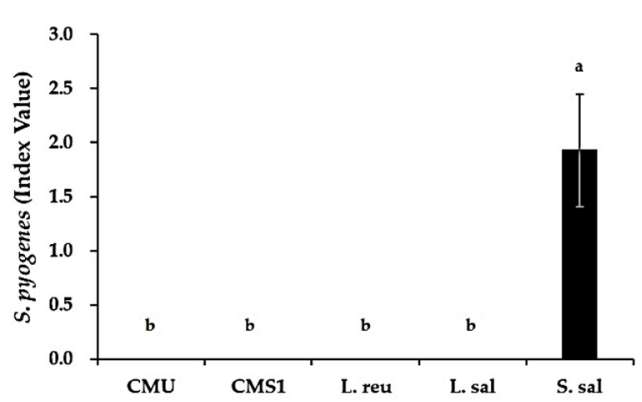

(c)

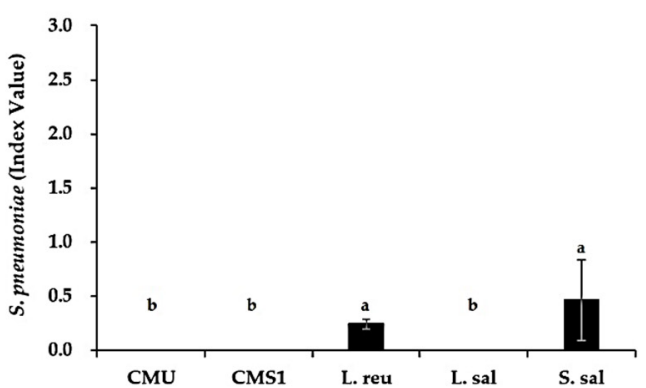

(b)

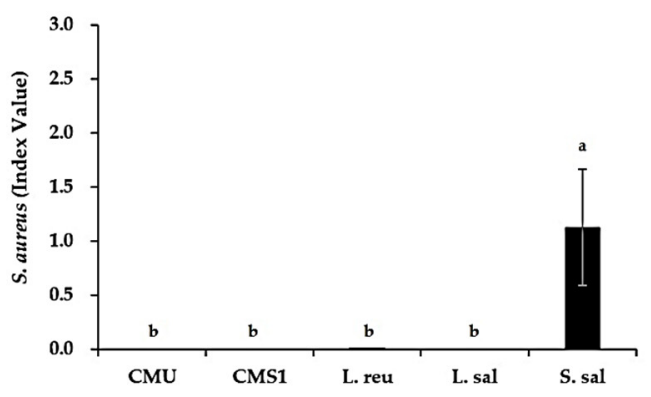

(d)

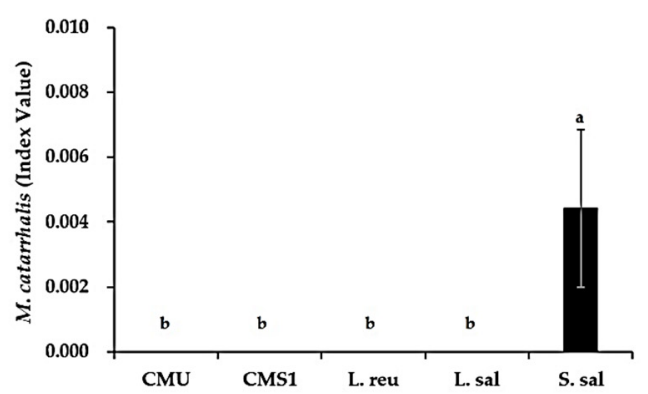

Figure 1. Competitive indexes (CIs) in coculture of oral care probiotics and major pathogens of URTIs. (a) Streptococcus pyogenes, (b) Staphylococcus aureus, (c) Streptococcus pneumoniae, and (d) Moraxella catarrhalis. Different letters (a and b) indicate significant differences at $p<0.05$. CMU, Weissella cibaria CMU; CMS1, W. cibaria CMS1; L. reu, Limosilactobacillus reuteri; L. sal, Ligilactobacillus salivarius; S. sal, Streptococcus salivarius.

Table 1. Minimum inhibitory concentrations (MICs) of cell-free supernatants of oral care probiotics against pathogens.

\begin{tabular}{ccccc}
\hline \multirow{2}{*}{ Probiotic } & \multicolumn{4}{c}{ MIC $(\mathbf{m g} / \mathbf{m L})$} \\
\cline { 2 - 5 } & S. pyogenes & Staph. aureus & S. pneumoniae & M. catarrhalis \\
\hline W. cibaria CMU & 250 & 125 & 125 & 125 \\
W. cibaria CMS1 & 250 & 125 & 125 & 125 \\
L. reuteri & 250 & 125 & 125 & 125 \\
L. salivarius & 125 & 125 & 125 & 125 \\
S. salivarius & $>500$ & $>500$ & $>500$ & $>500$
\end{tabular}

S. pyogenes, Streptococcus pyogenes; Staph. aureus, Staphylococcus aureus; S. pneumoniae, Streptococcus pneumoniae; M. catarrhalis, Moraxella catarrhalis; W. cibaria CMU, Weissella cibaria CMU; W. cibaria CMS1, Weissella cibaria CMS1; L. reuteri, Limosilactobacillus reuteri; L. salivarius, Ligilactobacillus salivarius; S. salivarius, Streptococcus salivarius.

\subsubsection{Time to Kill Major Pathogens of URTIs}

Figure 2 shows the killing time for CFSs of the $W$. cibaria strains against the four major pathogens. At $2 \times$ MIC, the CFSs of both $W$. cibaria strains showed complete bactericidal effects for S. pyogenes and M. catarrhalis within 4 and $6 \mathrm{~h}$, respectively, and for Staph. aureus and S. pneumoniae within $24 \mathrm{~h}$ of exposure. 
(a)
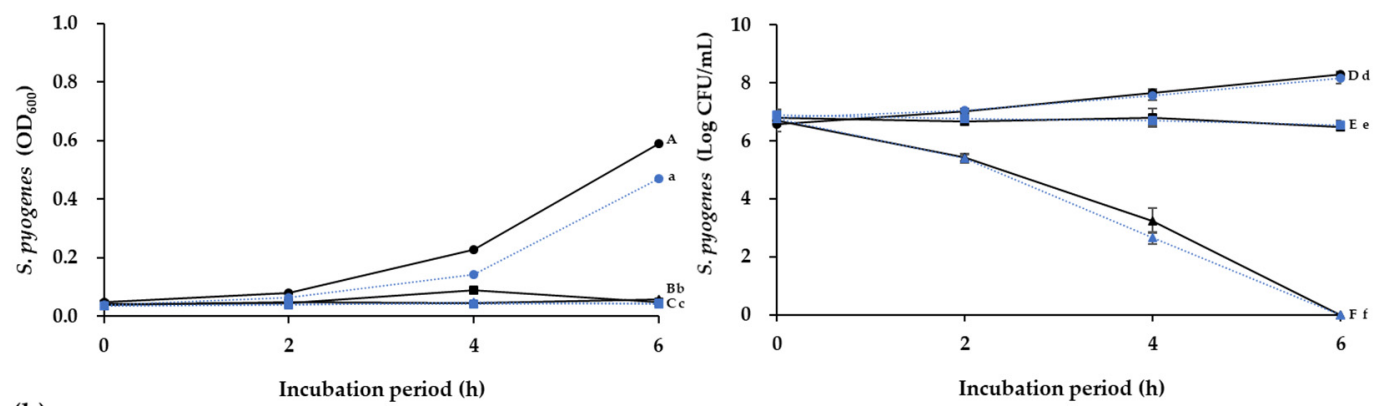

(b)
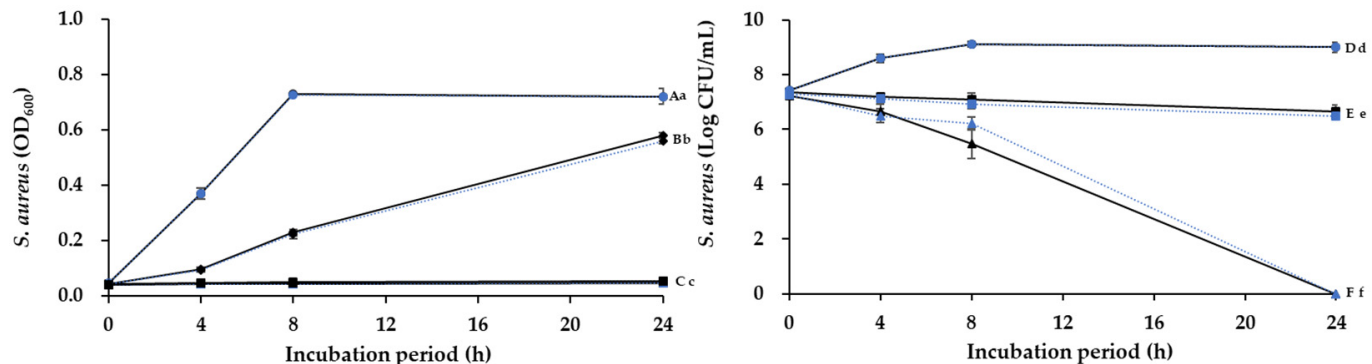

(c)
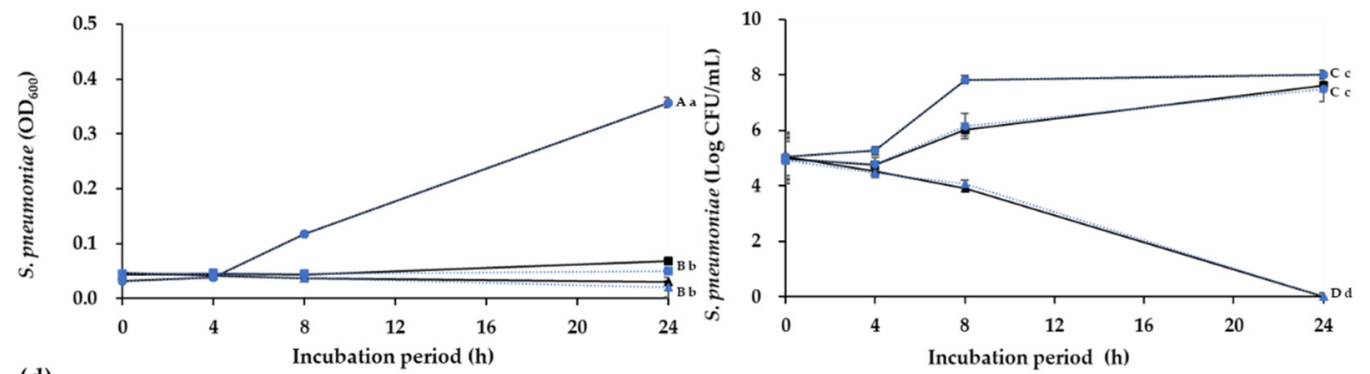

(d)
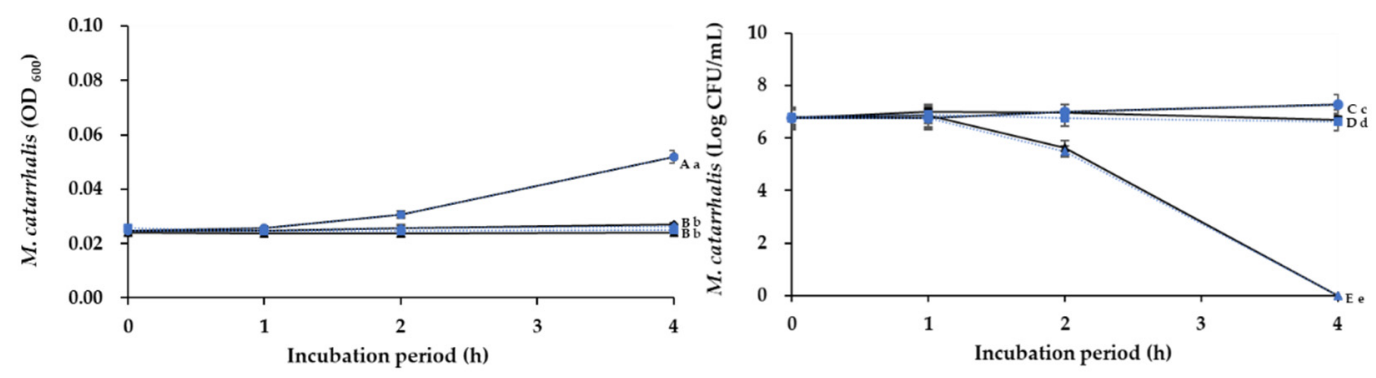

Figure 2. Time-kill curves for cell-free supernatants of Weissella cibaria strains against (a) Streptococcus pyogenes, (b) Staphylococcus aureus, (c) S. pneumoniae, and (d) Moraxella catarrhalis, at different minimum inhibitory concentration (MIC) increments: $\boldsymbol{\Lambda}, 2 \times \mathrm{MIC} ; \mathbf{\square}, 1 \times \mathrm{MIC} ; \bullet$, untreated control. Solid line, W. cibaria CMU (oraCMU); broken line, W. cibaria CMS1 (oraCMS1). Different letters (A-F) indicate significant differences among oraCMU treatment groups $(p<0.05)$. Different letters (a-f) indicate significant differences among oraCMS1 treatment groups $(p<0.05)$. $\mathrm{OD}_{600}$, absorbance at $600 \mathrm{~nm}$; CFU, colonyforming units.

\subsubsection{Characterization of Antimicrobial Substances}

The identification of antimicrobial substances of $W$. cibaria showed that only organic acids acted on Staph. aureus and S. pneumoniae. $\mathrm{H}_{2} \mathrm{O}_{2}$ showed a dose-dependent antimicrobial activity against $S$. pyogenes and $M$. catarrhalis, and BLCs only acted on $M$. catarrhalis (Figure 3). 
(a)

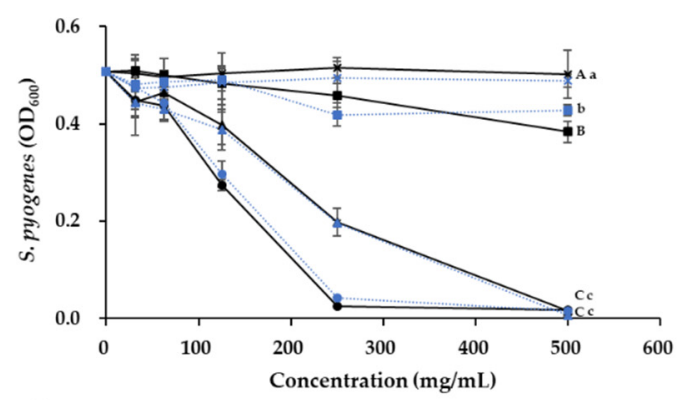

(c)

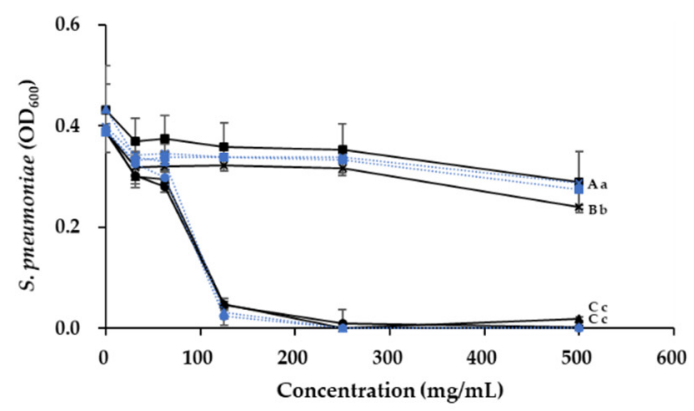

(b)

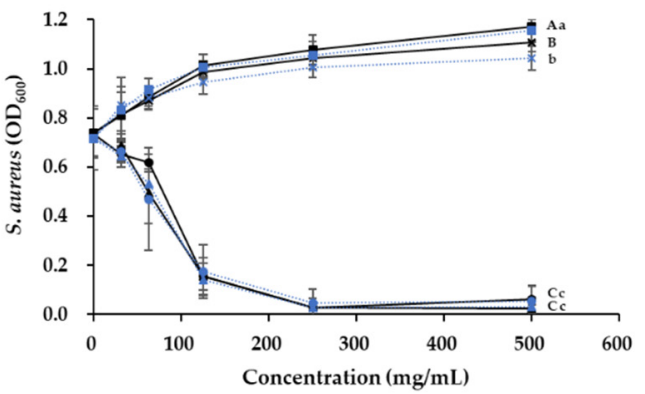

(d)

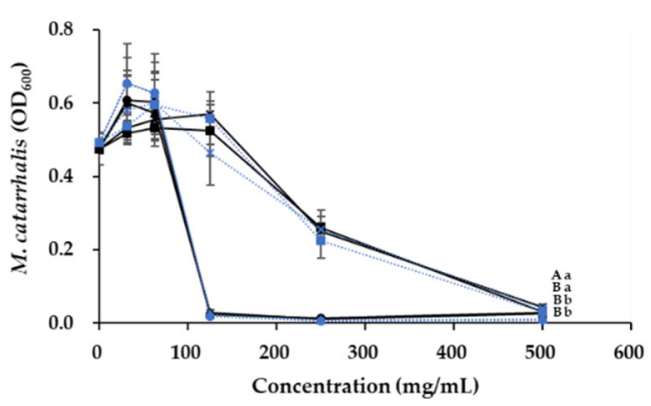

Figure 3. Dose-dependent effects of organic acids, $\mathrm{H}_{2} \mathrm{O}_{2}$, and bacteriocin-like compounds (BLCs) in cell-free supernatants (CFSs) of Weissella cibaria strains against (a) Streptococcus pyogenes, (b) Staphylococcus aureus, (c) S. pneumoniae, and (d) Moraxella catarrhalis. $\bullet, \mathrm{CFS} ; \boldsymbol{\Lambda}$, organic acids; $\mathbf{\square}, \mathrm{H}_{2} \mathrm{O}_{2} ; \mathrm{X}$, BLCs. Solid line, W. cibaria CMU (oraCMU); broken line, $W$. cibaria CMS1 (oraCMS1). Different letters (A-C) indicate significant differences among oraCMU treatment groups $(p<0.05)$. Different letters $(\mathrm{a}-\mathrm{c})$ indicate significant differences among oraCMS1 treatment groups $(p<0.05)$. OD 600 , absorbance at $600 \mathrm{~nm}$.

\subsection{Antibiofilm Activity}

\subsubsection{Inhibition of Biofilm Formation}

Both live cells and CFSs of the W. cibaria strains showed similar inhibitory effects on biofilm formation by the four major pathogens of URTIs (Figure 4). After $48 \mathrm{~h}$ of incubation, live cells of the $W$. cibaria strains significantly reduced biofilm formation by the pathogens (S. pyogenes, 60-62\%; Staph. aureus, 68-76\%; S. pneumonia, 56-62\%; and M. catarrhalis, $54-59 \%)(p<0.05)$. The CFSs of the $W$. cibaria strains also significantly reduced biofilm formation by S. pyogenes (80-86\%), Staph. aureus (92-93\%), S. pneumoniae (73-74\%), and M. catarrhalis (56-61\%).

\subsubsection{Removal of Preformed Biofilms}

Both live bacteria and CFSs of the W. cibaria strains showed similarly excellent biofilm removal abilities for all major pathogens of URTIs, except S. pneumonia (Figure 5), with the best removal effect observed against the Staph. aureus biofilm.

\subsubsection{CLSM Results}

CLSM analysis was used to observe the effects of live cells and CFSs of the W. cibaria strains on S. pyogenes biofilm formation. Very small amounts of biofilm were observed in all areas treated with live cells or CFSs of the W. cibaria strains compared to the positive control group (Figure 6a). In addition, the numbers of dead cells increased in the W. cibaria-treated groups (Figures $6 \mathrm{~b}$ and 7 ). 
(a)

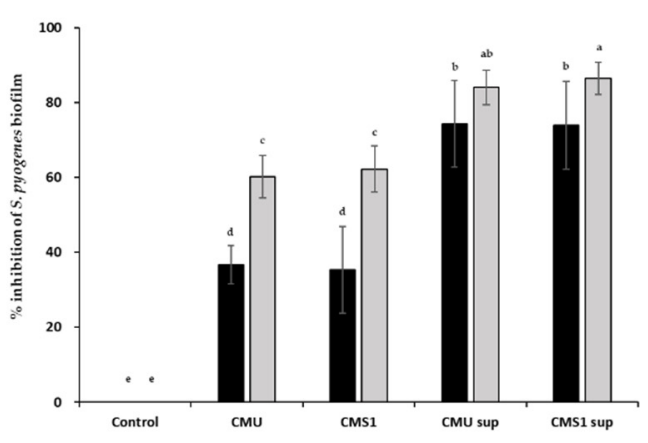

(c)

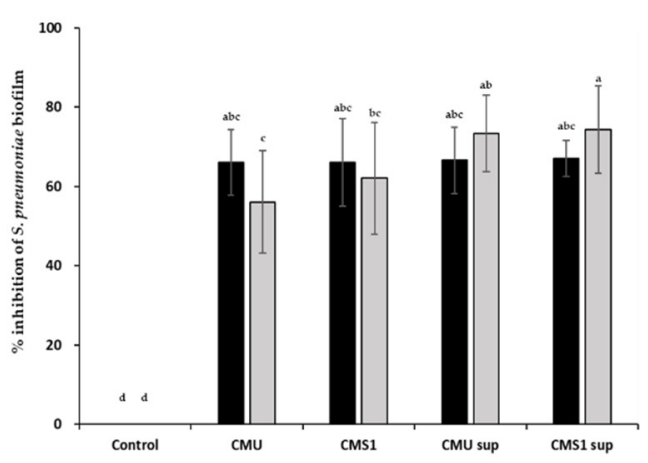

(b)

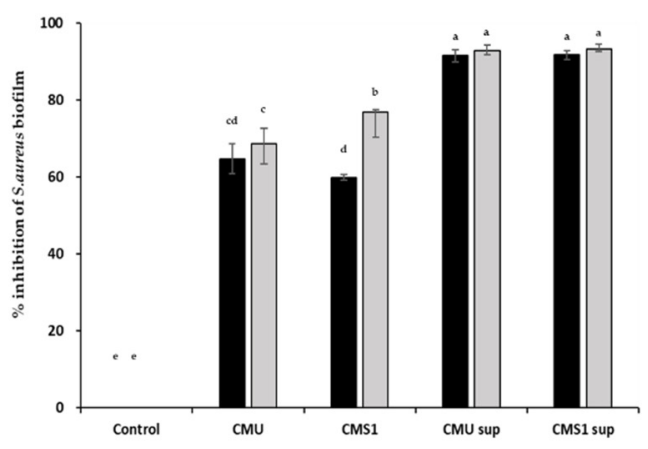

(d)

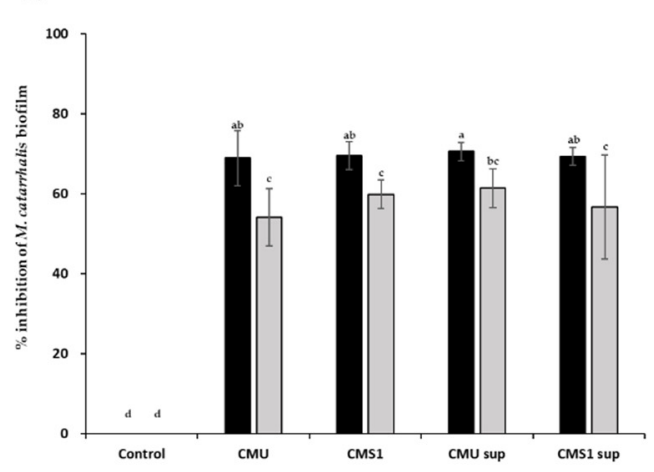

Figure 4. Inhibitory effects of Weissella cibaria strains on biofilm formation by (a) Streptococcus pyogenes, (b) Staphylococcus aureus, (c) S. pneumoniae, and (d) Moraxella catarrhalis. Black bar, $24 \mathrm{~h}$; gray bar, $48 \mathrm{~h}$. CMU, W. cibaria CMU; CMS1, W. cibaria CMS1; CMU sup, cell-free supernatant (CFS) of W. cibaria CMU; CMS1 sup, CFS of W. cibaria CMS1. Different letters (a-e) indicate significant differences at $p<0.05$.
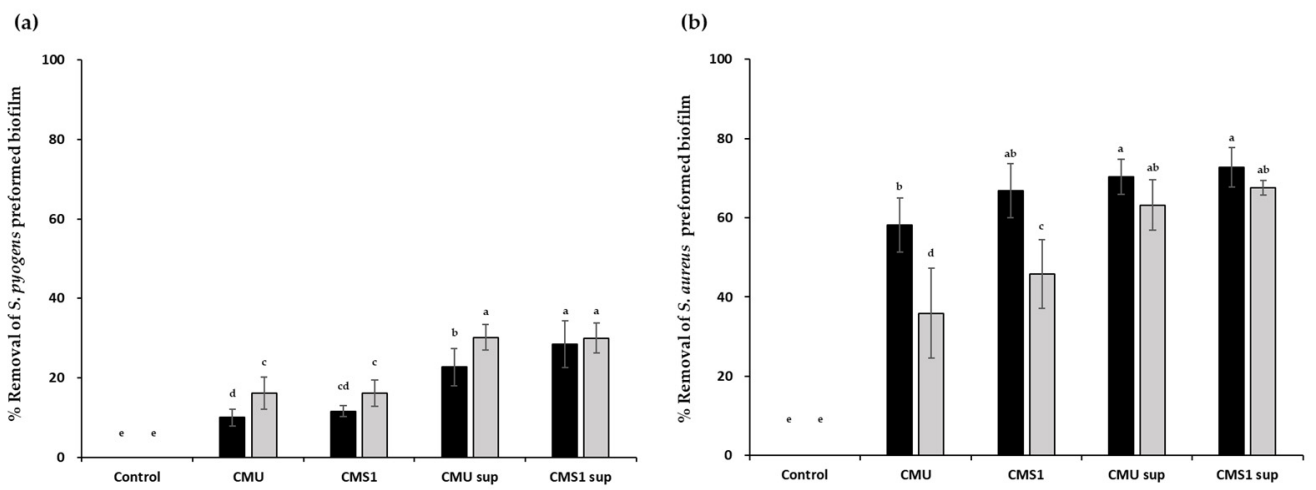

Figure 5. Cont. 

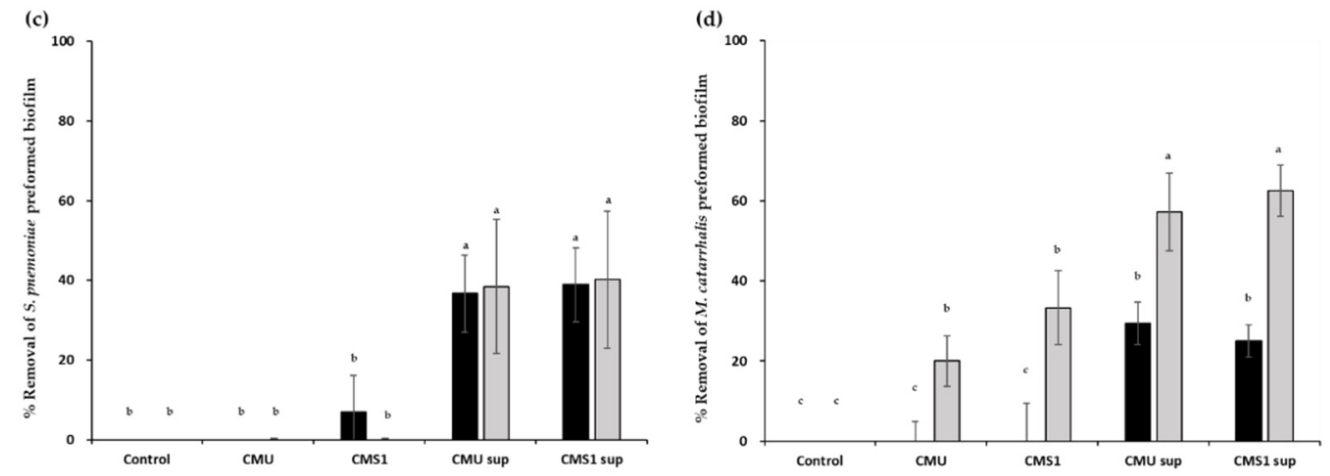

Figure 5. Removal effects of Weissella cibaria strains on preformed biofilms of (a) Streptococcus pyogenes, (b) Staphylococcus aureus, (c) S. pneumoniae, and (d) Moraxella catarrhalis. Black bar, $24 \mathrm{~h}$; gray bar, $48 \mathrm{~h}$. CMU, W. cibaria CMU; CMS1, W. cibaria CMS1; CMU sup, cell-free supernatant (CFS) of W. cibaria CMU; CMS1 sup, CFS of W. cibaria CMS1. Different letters (a-e) indicate significant differences at $p<0.05$.

(a)

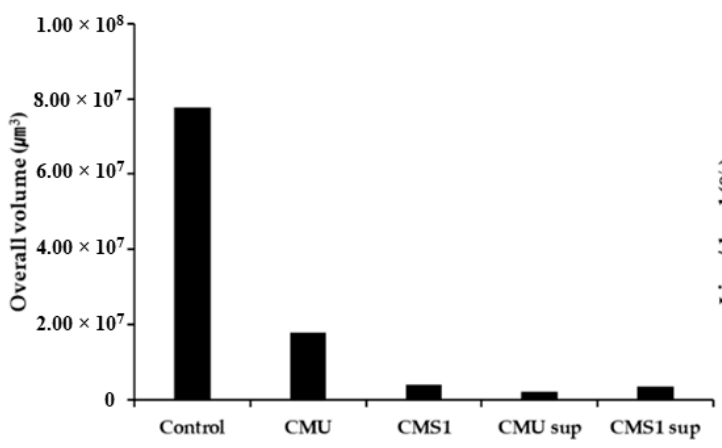

(b)

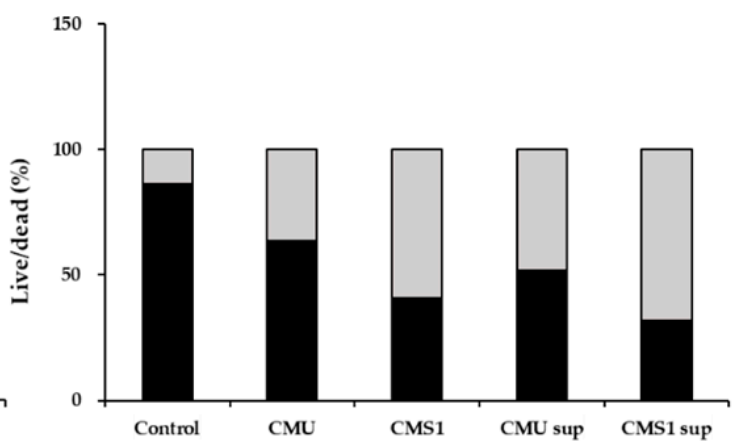

Figure 6. Results of confocal scanning microscopy analysis. (a) Overall biofilm volumes; (b) live/dead cell ratios. Black bar, live cells; gray bar, dead cells. CMU, Weissella cibaria CMU; CMS1, W. cibaria CMS1; CMU sup, cell-free supernatant (CFS) of W. cibaria CMU; CMS1 sup, CFS of W. cibaria CMS1.

(b)

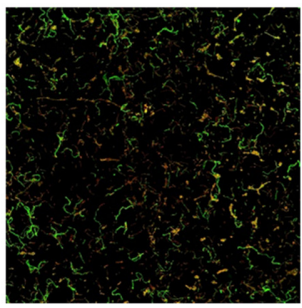

(d)

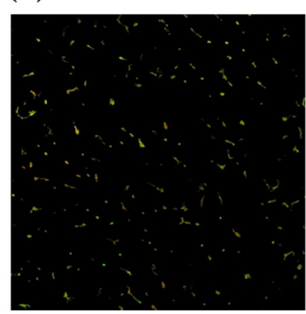

(c)

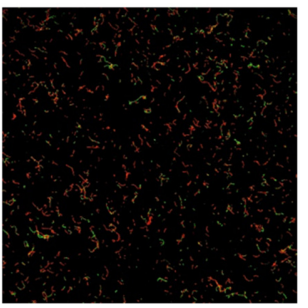

(e)

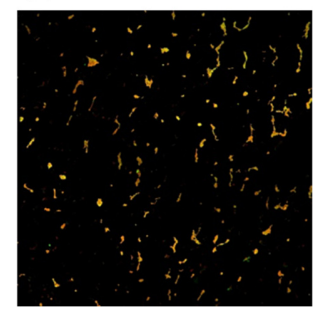

Figure 7. Confocal laser scanning microscopy images of Streptococcus pyogenes biofilms. (a) Untreated control; (b) treatment with live cells of Weissella cibaria CMU (oraCMU); (c) treatment with live cells of W. cibaria CMS1 (oraCMS1); (d) treatment with a cell-free supernatant (CFS) of oraCMU; (e) treatment with a CFS of oraCMS1. Green, live cells; red, dead cells (magnification, $40 \times$ ). 


\section{Discussion}

W. cibaria is a probiotic found in various fermented foods [25-28]. In particular, $W$. cibaria plays an important role in the maturation of kimchi, a traditional fermented food in Korea, and is a dominant species during the aging process of kimchi. oraCMU and oraCMS1 are W. cibaria strains isolated and identified from the saliva of 460 children aged 4-7 years with good oral health [29]. These bacteria have been reported to be effective in preventing oral diseases (bad breath, periodontal disease, and tooth decay) by reducing oral pathogens via antibacterial, antibiofilm, and co-aggregation effects [20].

Many previous studies of $W$. cibaria have focused on oral health $[20,30,31]$. We hypothesized that because the upper respiratory tract includes organs that are closest to the oral cavity, $W$. cibaria could affect URTIs. Therefore, in this study, W. cibaria was investigated in vitro to determine its potential for the prevention and treatment of URTIs. S. pneumoniae, S. pyogenes, and M. catarrhalis, the most common bacterial pathogens that cause acute otitis media and pharyngeal tonsillitis, and Staph. aureus, which is involved in refractory chronic rhinosinusitis [32-34], were selected as major pathogens.

In our study, the antimicrobial activities of $W$. cibaria strains against the major pathogens of URTIs were compared with those of other commercial oral care probiotics. CIs were calculated to determine the exact nature of the competitive ability of the species. The target pathogens exhibited CIs below 1 , which indicated their poor competition in coculture with the oral care probiotics. Except for S. salivarius, which showed poor competition with S. pyogenes and Staph. aureus, all other tested oral care probiotics competed well. The antimicrobial activity of $S$. salivarius was the lowest against the four pathogens tested. It was also confirmed that S. pneumoniae and M. catarrhalis did not compete well with any oral care probiotic. As a result, except for S. salivarius, both live cells and CFSs of the other oral care probiotics showed similar and remarkable antimicrobial activities against all pathogens.

S. salivarius is known to show antimicrobial activity against S. pyogenes, a sore throatcausing bacterium. However, according to Fiedler et al. [35], S. salivarius did not kill $S$. pyogenes at a concentration similar to that of $S$. pyogenes, which was consistent with the failure of $S$. salivarius to kill S. pyogenes in this study. In addition, S. salivarius did not show any antimicrobial activity against the other pathogens. Meanwhile, the CFS of L. salivarius showed the best antimicrobial activity, with a MIC of $125 \mathrm{mg} / \mathrm{mL}$ for S. pyogenes, and live bacteria demonstrated excellent competitive inhibition of the pathogens. These findings were consistent with the data of a previous study in which L. salivarius completely killed Staph. aureus in coculture [36].

The results of the time-kill assay were useful to determine against which of the four major pathogens of URTIs the bactericidal properties of the $W$. cibaria strains were most effective. The results showed that both oraCMU and oraCMS1 were most effective in killing M. catarrhalis within $4 \mathrm{~h}$ at a CFS concentration of $2 \times$ MIC, followed by killing S. pyogenes within $6 \mathrm{~h}$, while Staph. aureus and S. pneumoniae were completely killed within $24 \mathrm{~h}$.

A previous study showed that the CFS of S. pneumoniae inhibited the growth of $H$. influenzae, whereas the CFS of $H$. influenzae did not affect the growth of S. pneumoniae [37]. Furthermore, $\mathrm{H}$. influenzae was reported to be killed by $\mathrm{H}_{2} \mathrm{O}_{2}$ produced by $S$. pneumoniae, and a reversible inhibitory effect of $S$. pneumoniae was also observed on the growth of M. catarrhalis. These data support our findings that $S$. pneumoniae was not affected by $\mathrm{H}_{2} \mathrm{O}_{2}$ produced by $W$. cibaria, while M. catarrhalis was affected. In addition, Staph. aureus was not affected by $\mathrm{H}_{2} \mathrm{O}_{2}$ produced by the $W$. cibaria strains, likely because the catalase produced by Staph. aureus [38] eliminated the effects of $\mathrm{H}_{2} \mathrm{O}_{2}$. Therefore, it was confirmed that the $W$. cibaria strains had stronger antimicrobial activities when antimicrobial substances such as organic acids, $\mathrm{H}_{2} \mathrm{O}_{2}$, and BLCs acted together, as was observed in the case of M. catarrhalis.

Biofilm formation, which is due to the secretion of extracellular polymeric materials, provides stability to microbial populations by allowing bacteria to adhere to surfaces. In 
this state, an initial biofilm is formed when bacterial cells form a colony. In the process of biofilm growth and maturation, bacterial cells are released, and free-floating bacteria are disseminated to cause infection in surrounding tissues or other organs [39].

Biofilms ensure good survival and protection of pathogens from host defense mechanisms, antibiotics, and other environmental factors [40]. Therefore, once a biofilm is formed, it is difficult to eradicate, even with conventional antibiotic treatment [41]. Moreover, it is well known that the use of antibiotics not only does not reduce complications of bacterial infections, but increases medical costs by causing side effects and antibiotic resistance [3-5]. Biofilm formation by pathogens of URTIs can be an important cause of chronic infectious diseases of the upper respiratory tract, including recurrent middle ear disease, chronic rhinosinusitis, and recurrent pharyngeal tonsillitis [41]. Therefore, it is most effective to prevent pathogens from forming a biofilm, for which new treatments are needed.

In the present study, the W. cibaria strains were tested for their ability to affect biofilm formation by the four major pathogens of URTIs. In a Transwell assay, in which live cells of the W. cibaria strains were not directly cultured with the target pathogen, inhibition of pathogenic biofilm formation was observed. In addition, both live cells and CFSs of the $W$. cibaria strains showed the ability to facilitate biofilm removal, although they were more effective in inhibiting biofilm formation than in removing preformed biofilms. These results suggest that the antibiofilm activities of the $W$. cibaria strains are due to secreted substances and do not require direct contact with pathogens.

Similar to the results of Bidossi et al. [42], W. cibaria strains inhibited biofilm formation by S. pneumoniae, M. catarrhalis, and Staph. aureus. In addition, biofilm formation by S. pyogenes, which is not significantly affected by the presence of probiotic strains, was also inhibited, and the biofilm was removed. Wang et al. [43] reported that $W$. cibaria FbpA prevented Staph. aureus colonization by interfering with the invasion pathway and inhibiting biofilm formation. Similarly, in our study, the W. cibaria strains were most effective in inhibiting biofilm formation and removing biofilm of Staph. aureus, among the four target pathogens.

URTIs are mainly caused by viruses [2], but the prevention and treatment of infections caused by certain bacterial pathogens are also important. To the best of our knowledge, this study is the first to confirm the preventive effects of $W$. cibaria on URTIs in vitro. The results indicated that both $W$. cibaria probiotic strains had desirable functions to be used against pathogens of URTIs.

Substances that can regulate the expression of protein mediators of inflammatory responses and signaling pathways are known to play an important role in the prevention and treatment of URTIs $[14,44]$; therefore, further studies are needed.

\section{Conclusions}

This study reports the antimicrobial activities of $W$. cibaria oral care probiotics against major pathogens of URTIs, including the inhibition of biofilm formation. These findings suggest the potential of $W$. cibaria to be used as an alternative prevention and treatment agent in the management of URTIs.

Author Contributions: Conceptualization, J.-E.Y., M.-S.K. and H.-G.L.; formal analysis, J.-E.Y. and J.L.; funding acquisition, M.-S.K.; investigation, J.-E.Y., G.-Y.P. and J.L.; methodology, M.-S.K., J.-E.Y. and G.-Y.P.; project administration, J.-E.Y. and M.-S.K.; writing-original draft, J.-E.Y.; writingreview and editing, J.-E.Y., M.-S.K. and H.-G.L. All authors have read and agreed to the published version of the manuscript.

Funding: This work was supported by the Korea Institute of Planning and Evaluation for Technology in Food, Agriculture, Forestry (IPET) through the Useful Agricultural Life Resources Industry Technology Development Program, funded by the Ministry of Agriculture, Food and Rural Affairs (MAFRA) (No. 120050-2), and by a Basic Science Research Program of the National Research Foundation of Korea grant (No. 2020R1F1A1069551), funded by the Ministry of Science and ICT (MSIT) of Korea. 
Institutional Review Board Statement: Not applicable.

Informed Consent Statement: Not applicable.

Data Availability Statement: The data presented in this study are available on request from the corresponding author.

Conflicts of Interest: J.-E.Y., G.-Y.P. and M.-S.K. are employees of OraPharm, Inc. The other authors report no competing financial interests.

\section{References}

1. Jain, N.; Lodha, R.; Kabra, S.K. Upper respiratory tract infections. Indian J. Pediatr. 2001, 68, 1135-1138. [CrossRef]

2. Grief, S.N. Upper respiratory infections. Prim. Care 2013, 40, 757-770. [CrossRef]

3. Johnston, S.; Holgate, S. Epidemiology of viral respiratory infections. In Viral and Other Infections of the Human Respiratory Tract; Myint, S., Taylor-Robinson, D., Eds.; Springer: Dordrecht, The Netherlands, 1996; ISBN 978-94-011-7930-0.

4. Fendrick, M.A.; Monto, S.A.; Nightengale, B.; Sarnes, M. The economic burden of non-influenza-related viral respiratory tract infection in the United States. Arch. Intern. Med. 2003, 163, 487-494. [CrossRef] [PubMed]

5. Kirkpatrick, G.L. The common cold. Prim. Care 1996, 23, 657-675. [CrossRef]

6. Richard, R.M.; David, A.; Neil, B.; Dickson, C.; Steven, E.; Theodore, G.G.; Andrea, G.; Daniel, H.; Richard, C.H., III; Patricia, A.H.; et al. Clinical practice guideline: Adult sinusitis. Otolaryngol. Head Neck Surg. 2007, 137, S1-S31.

7. Matthys, J.; De Meyere, M.; van Driel, M.L.; De Sutter, A. Differences among international pharyngitis guidelines: Not just academic. Ann. Fam. Med. 2007, 5, 436-443. [CrossRef] [PubMed]

8. Reveiz, L.; Cardona, A.F.; Ospina, E.G. Antibiotics for acute laryngitis in adults. Cochrane Database Syst. Rev. 2013, 28, CD004783.

9. Brook, I. Anaerobic bacteria in upper respiratory tract and head and neck infections: Microbiology and treatment. Anaerobe 2012, 18, 214-220. [CrossRef]

10. Pettigrew, M.M.; Gent, J.F.; Pyles, R.B.; Miller, A.L.; Nokso-Koivisto, J.; Chonmaitree, T. Viral-bacterial interactions and risk of acute otitis media complicating upper respiratory tract infection. J. Clin. Microbiol. 2011, 49, 3750-3755. [CrossRef]

11. Li, J.; Song, X.; Yang, T.; Chen, Y.; Gong, Y.; Yin, X.; Lu, Z. A systematic review of antibiotic prescription associated with upper respiratory tract infections in China. Medicine 2016, 95, e3587. [CrossRef] [PubMed]

12. Martin, J.M. The Mysteries of streptococcal pharyngitis. Curr. Treat Options Pediatr. 2015, 1, 180-189. [CrossRef]

13. Costerton, J.W.; Lewandowski, Z.; Caldwell, D.E.; Korber, D.R.; LappinScott, H.M. Microbial biofilms. Annu. Rev. Microbiol. 1995, 49, 711-745. [CrossRef]

14. Singh, V.P.; Sharma, J.; Babu, S.; Rizwanulla, S.A.; Singla, A. Role of probiotics in health and disease: A review. J. Pak. Med. Assoc. 2003, 63, 253-257.

15. Altadill, T.; Espadaler-Mazo, J.; Liong, M.T. Effects of a Lactobacilli probiotic on reducing duration of URTI and fever, and use of URTI-associated medicine: A re-analysis of a randomized, placebo-controlled study. Microorganisms 2021, 9, 528. [CrossRef]

16. Di Pierro, F.; Risso, P.; Poggi, E.; Timitilli, A.; Bolloli, S.; Bruno, M.; Caneva, E.; Campus, R.; Giannattasio, A. Use of Streptococcus salivarius $\mathrm{K} 12$ to reduce the incidence of pharyngo-tonsillitis and acute otitis media in children: A retrospective analysis in not-recurrent pediatric subjects. Minerva Pediatr. 2018, 70, 240-245. [PubMed]

17. Kang, M.S.; Yeu, J.E.; Hong, S.P. Safety evaluation of oral care probiotics Weissella cibaria CMU and CMS1 by phenotypic and genotypic analysis. Int. J. Mol. Sci. 2019, 20, 2693. [CrossRef] [PubMed]

18. Lee, D.S.; Lee, S.A.; Kim, M.; Nam, S.H.; Kang, M.S. Reduction of halitosis by a tablet containing Weissella cibaria CMU: A randomized, double-blind, placebo- controlled study. J. Med. Food. 2020, 23, 649-657. [CrossRef]

19. Do, K.H.; Park, H.E.; Kang, M.S.; Kim, J.T.; Yeu, J.E.; Lee, W.K. Effects of Weissella cibaria CMU on halitosis and calculus, plaque, and gingivitis indices in beagles. J. Vet. Dent. 2019, 36, 135-142. [CrossRef]

20. Jang, H.J.; Kang, M.S.; Yi, S.H.; Hong, J.Y.; Hong, S.P. Comparative study on the characteristics of Weissella cibaria CMU and probiotic strains for oral care. Molecules 2016, 21, 1752. [CrossRef] [PubMed]

21. Birkenhauer, E.; Neethirajan, S. Characterization of electrical surface properties of mono- and co-cultures of Pseudomonas aeruginosa and methicillin-resistant Staphylococcus aureus using Kelvin probe force microscopy. RSC. Adv. 2014, 4, 42432-42440. [CrossRef]

22. Wijesundara, N.M.; Rupasinghe, H. Bactericidal and anti-biofilm activity of ethanol extracts derived from selected medicinal plants against Streptococcus pyogenes. Molecules 2019, 24, 1165. [CrossRef] [PubMed]

23. Wijesundara, N.M.; Rupasinghe, H. Essential oils from Origanum vulgare and Salvia officinalis exhibit antibacterial and anti-biofilm activities against Streptococcus pyogenes. Microb. Pathog. 2018, 117, 118-127. [CrossRef] [PubMed]

24. Kang, M.S.; Oh, J.S.; Lee, H.C.; Lim, H.S.; Lee, S.W.; Yang, K.H.; Choi, N.K.; Kim, S.M. Inhibitory effect of Lactobacillus reuteri on periodontopathic and cariogenic bacteria. J. Microbiol. 2011, 49, 193-199. [CrossRef] [PubMed]

25. Kim, J.H.; Lee, K.W.; Han, N.S.; Park, J.Y.; Chun, J.Y. Importance of Weissella species during kimchi fermentation and future works. Arch. Intern. Med. 2003, 163, 487-494.

26. Chen, Y.S.; Wu, H.C.; Lo, H.Y.; Lin, W.C.; Hsu, W.H.; Lin, C.W.; Lin, P.Y.; Yanagida, F. Isolation and characterisation of lactic acid bacteria from jiang-gua (fermented cucumbers), a traditional fermented food in Taiwan. J. Sci. Food Agric. 2012, 92, $2069-2075$. [CrossRef] [PubMed] 
27. González-Quijano, G.K.; Dorantes-Alvarez, L.; Hernández-Sánchez, H.; Jaramillo-Flores, M.E.; de Jesús Perea-Flores, M.; VeraPonce de León, A.; Hernández-Rodríguez, C. Halotolerance and survival kinetics of lactic acid bacteria isolated from jalapeño pepper (Capsicum annuum L.) fermentation. J. Food Sci. 2014, 79, M1545-M1553. [CrossRef]

28. Scheirlinck, I.; Van der Meulen, R.; Van Schoor, A.; Vancanneyt, M.; De Vuyst, L.; Vandamme, P.; Huys, G. Influence of geographical origin and flour type on diversity of lactic acid bacteria in traditional Belgian sourdoughs. Appl. Environ. Microbiol. 2007, 73, 6262-6269. [CrossRef]

29. Kang, M.S.; Kim, B.G.; Chung, J.; Lee, H.C.; Oh, J.S. Inhibitory effect of Weissella cibaria isolates on the production of volatile sulphur compounds. J. Clin. Periodontol. 2006, 33, 226-232. [CrossRef]

30. Kang, M.S.; Chung, J.; Kim, S.M.; Yang, K.H.; Oh, J.S. Effect of Weissella cibaria isolates on the formation of Streptococcus mutans biofilm. Caries Res. 2006, 40, 418-425. [CrossRef]

31. Kim, H.J.; Yeu, J.E.; Lee, D.S.; Kang, M.S. Weissella cibaria CMU suppresses $m g l$ gene expression and enzyme activity associated with bad breath. Int. J. Oral Biol. 2019, 44, 152-159. [CrossRef]

32. Worrall, G. Acute otitis media. Can. Fam. Physician 2007, 53, 2147-2148. [PubMed]

33. Murray, R.C.; Chennupati, S.K. Chronic streptococcal and non-streptococcal pharyngitis. Infect. Disord. Drug Targets 2012, 12, 281-285. [CrossRef] [PubMed]

34. Foreman, A.; Boase, S.; Psaltis, A.; Wormald, P.J. Role of bacterial and fungal biofilms in chronic rhinosinusitis. Curr. Allergy Asthma Rep. 2012, 12, 127-135. [CrossRef] [PubMed]

35. Fiedler, T.; Riani, C.; Koczan, D.; Standar, K.; Kreikemeyer, B.; Podbielski, A. Protective mechanisms of respiratory tract Streptococci against Streptococcus pyogenes biofilm formation and epithelial cell infection. Appl. Environ. Microbiol. 2013, 79, 1265-1276. [CrossRef]

36. Kang, M.S.; Lim, H.S.; Oh, J.S.; Lim, Y.J.; Wuertz-Kozak, K.; Harro, J.M.; Shirtliff, M.E.; Achermann, Y. Antimicrobial activity of Lactobacillus salivarius and Lactobacillus fermentum against Staphylococcus aureus. Pathog. Dis. 2017, 75, ftx009. [CrossRef]

37. Pericone, C.D.; Overweg, K.; Hermans, P.W.; Weiser, J.N. Inhibitory and bactericidal effects of hydrogen peroxide production by Streptococcus pneumoniae on other inhabitants of the upper respiratory tract. Infect. Immun. 2000, 68, 3990-3997. [CrossRef] [PubMed]

38. Mustafa, H. Staphylococcus aureus can produce catalase enzyme when adding to human WBCs as a source of $\mathrm{H} 2 \mathrm{O} 2$ productions in human plasma or serum in the laboratory. Indian J. Med. Microbiol. 2014, 4, 249-251.

39. Tolker-Nielsen, T. Biofilm Development. Microbiol. Spectr. 2015, 3. [CrossRef]

40. Ogawa, T.; Terao, Y.; Okuni, H.; Ninomiya, K.; Sakata, H.; Ikebe, K.; Maeda, Y.; Kawabata, S. Biofilm formation or internalization into epithelial cells enable Streptococcus pyogenes to evade antibiotic eradication in patients with pharyngitis. Microb. Pathog. 2011, 51, 58-68. [CrossRef]

41. Nazzari, E.; Torretta, S.; Pignataro, L.; Marchisio, P.; Esposito, S. Role of biofilm in children with recurrent upper respiratory tract infections. Eur. J. Clin. Microbiol. Infect. Dis. 2015, 34, 421-429. [CrossRef]

42. Bidossi, A.; De Grandi, R.; Toscano, M.; Bottagisio, M.; De Vecchi, E.; Gelardi, M.; Drago, L. Probiotics Streptococcus salivarius $24 \mathrm{SMB}$ and Streptococcus oralis 89 a interfere with biofilm formation of pathogens of the upper respiratory tract. BMC Infect. Dis. 2018, 18, 653. [CrossRef] [PubMed]

43. Wang, L.; Si, W.; Xue, H.; Zhao, X. A fibronectin-binding protein (FbpA) of Weissella cibaria inhibits colonization and infection of Staphylococcus aureus in mammary glands. Cell. Microbiol. 2017, 19, 10. [CrossRef] [PubMed]

44. Soderholm, A.T.; Barnett, T.C.; Sweet, M.J.; Walker, M.J. Group A streptococcal pharyngitis: Immune responses involved in bacterial clearance and GAS-associated immunopathologies. J. Leukoc. Biol. 2018, 103, 193-213. [CrossRef] [PubMed] 